\title{
Rectal bleeding in the first month of life
}

\author{
MALCOLM I. LeVENE \\ M.B., B.S.(Lond.), M.R.C.S.(Eng.), L.R.C.P.(Lond.) \\ Derby Children's Hospital, North Street, Derby DE1 3BA
}

\begin{abstract}
Summary
Sixty-nine infants were examined who presented with rectal bleeding in the first month of life. In $64 \%$ of these children no cause was found. Infantile diarrhoea, haemorrhagic disease of the newborn and anal fissure were the most common diagnoses made. The mortality in the entire group was $<3 \%$. It is concluded that rectal bleeding in this age group is a relatively benign condition and in certain cases hospital admission may not be required.
\end{abstract}

\section{Introduction}

Fresh bleeding from the rectum is an alarming event for the parents of a young infant, and often poses a dilemma to the doctor as to the referral and extent of investigation of his patient. This study was undertaken to give the experience of this condition in a paediatric hospital over the last 20 years.

\section{Method and results}

The records of all children presenting at this hospital in the first month of life with any of the diagnoses shown in Table 1 were examined. Derby Children's Hospital serves an area of approximately 500000 people and takes all the paediatric medical and surgical referrals in the area. A total of $\mathbf{3 1 2}$ such children were seen between 1958 and 1977, of whom 84 had been originally referred, either to the physician or a surgeon, with rectal bleeding as the presenting problem. Infants who developed rectal bleeding in the Special Care Baby Unit, or in a nursing home were excluded from the study.

Some infants born before January 1972, when prophylactic vitamin $\mathrm{K}$ was introduced, were given this substance on presentation with bleeding before a prothrombin time was performed. These infants in whom the diagnosis of haemorrhagic disease of the newborn could not be substantiated by a prothrombin time were also excluded from the study. Of the original 84 infants who fulfilled the original criteria, a further 15 were withdrawn owing to inadequate investigation. A total of 69 infants is included in this study.

Present address: Department of Paediatrics, Charing Cross Hospital, London W6 8RF.
Bleeding was noted in the first 7 days of life in 32 infants $(46 \%)$, with the fifth day being the commonest day of onset. Ten $(14 \%)$ first bled in the third week, and $8(12 \%)$ in the fourth week of life. Sixty per cent of the total group were male.

In $15 \%$, blood was noted on less than 3 occasions and was usually only a few specks in the stool. Recurrent bleeding was noticed by the parents on more than three occasions in $67 \%$. This was generally streaky and mixed with the stool. In $14 \%$ there was a large amount of blood mixed with the stool or frank blood was passed per rectum.

TABLE 1.

\begin{tabular}{lc}
\hline \multicolumn{1}{c}{ Possible diagnoses } & Number of patient \\
\hline Anal fissure & 6 \\
Rectal prolapse/rectal polyps & 0 \\
Meckel's diverticulum & 0 \\
Bowel duplication & 0 \\
Intussusception & 1 \\
Necrotizing enterocolitis & 2 \\
Ulcerative colitis/Crohn's disease & 0 \\
Infantile diarrhoea & 9 \\
Acute gastric ulcer & 0 \\
Haemophilia/Christmas disease & 0 \\
Haemorrhagic disease of the newborn & 6 \\
von Willebrand's disease & 0 \\
Thrombocytopenia & 1 \\
Schönlein-Henoch purpura & 0 \\
Rectal bleeding - no cause found & 44 \\
& $\quad$ Total
\end{tabular}

Only $10 \%$ of infants were solely breast fed but, as the incidence of breast feeding has varied over the last 20 years, the significance of this is doubtful. The alarm that this symptom provoked is reflected in the time of presentation to hospital. Fifty-one infants $(73 \%)$ presented within $24 \mathrm{hr}$ of blood first being noted and only $4 \%$ of children were seen more than 5 days after the onset of bleeding. Two children were referred to out-patients.

Examination of the infant in general did not contribute to the diagnosis. In one infant generalized purpura was present and forearm abnormalities were obvious on inspection. Examination of the anus revealed fissures in 6 children, and a previous 
rectal examination was thought to have been the cause in at least 2 of these cases. In one child the diagnosis of intussusception was confirmed on rectal examination.

The commonest diagnosis was rectal bleeding associated with diarrhoea but no bacterial pathogens were isolated from any of these children. The diarrhoea was rarely severe, and only 2 infants required intravenous rehydration. Six infants had a prolonged prothrombin time and, in all, bleeding stopped after one injection of vitamin K. An anal fissure was found in 6 infants and was thought to be the cause of the rectal bleeding. Two children had necrotizing enterocolitis and one had intussusception. These 3 infants all appeared to be ill on presentation, and there was no difficulty in diagnosing an acute abdomen.

In 44 infants no cause was found and all stopped bleeding spontaneously; however, 9 continued to have fresh blood in the stool for more than 5 days, and one continued intermittently for a further 22 days. None required blood transfusion and apart from haematological investigation and careful examination to exclude a local lesion, no further investigations were performed.

Thirty-seven $(53 \%)$ of the infants presented after the end of the first week of life and before the end of the first month. No cause was found in 31 of these children, one had an anal fissure, and in 5 there was associated diarrhoea. None of these infants required any treatment and there were no deaths in this group.

Only 2 of the 69 children died. In one, death was attributable to uncontrollable bleeding into the bowel and brain due to thrombocytopenia with absent radii syndrome. The other death occurred postoperatively after laparotomy for malrotation of the bowel 4 days after original presentation with rectal bleeding associated with a prolonged prothrombin time.

In the 6 years from 1972 to 1977,34 infants were seen with rectal bleeding, and this represents $50 \%$ of the total over 20 years. In the last 5 years the numbers have been steady at about 5 infants requiring admission per year. This represents an incidence of $1 \cdot 5 / 10^{3} /$ year in the Derby area.

\section{Comment}

Although there are no specific references to rectal bleeding in the literature, Sherman and Clatworthy (1967) reported the study of 94 cases of gastrointestinal haemorrhage in neonates. Only 31 of those presented solely with fresh blood per rectum, and no cause was found for the bleeding in $52 \%$ of the total. All infants who developed rectal bleeding in the Special Care Baby Unit were excluded from the present trial, and this would account for the paucity of congenital gastrointestinal anomalies that may be associated with rectal bleeding.

It appears that bright red blood from the rectum in the first month of life is a condition which is generally self-limiting and requires no treatment. There is however a small group of children who have serious and potentially fatal surgical or haematological disease but these children will appear ill on presentation to hospital. Those infants presenting after the first week, and those associated with mild diarrhoea run a benign course, and probably only require observation. The condition is self-limiting and the parents should be reassured accordingly. All such infants require a thorough physical examination and a local cause carefully excluded.

The results of this study suggest that infants presenting with rectal bleeding in the first week of life require referral to hospital for investigation. This should include at least a haemoglobin and prothrombin time. Those children who have surgical conditions should pose no diagnostic problems.

\section{Reference}

Sherman, N.J. \& Clatworthy, H.W. (1967) Gastrointestinal bleeding in neonates. Surgery, 62, 614. 